Caries Res 2010;44:170

DOI: $\underline{10.1159 / 000314069}$

\section{Xylitol as Caries Prevention?}

\section{Dawes}

Department of Oral Biology, Faculty of Dentistry, University of Manitoba, Winnipeg, Man., Canada

I am responding to the article by Mäkinen [2009] in which he lauds the benefits, for caries prevention, of using products containing xylitol, compared with products containing other nonacidogenic polyols such as sorbitol. I was surprised that he did not reference the review article by van Loveren [2004], in which he concluded firstly that 2 out of the 4 clinical trials which compared the effects of xylitol and sorbitol did not confirm such a differential benefit of xylitol and secondly that there was no evidence for the caries-therapeutic effect of xylitol.

Although short-term studies on the comparative effects of products containing different polyols on the microbiology of dental plaque or saliva or on their ability to promote in situ remineralization of artificial white-spot lesions in enamel slabs may be of some interest, the only truly relevant test is a proper clinical trial. As such clinical caries trials take at least 2 years, I do not think that Professor Mäkinen need worry about possible problems with crossover studies.

Of course, if one agrees with Professor Mäkinen's belief that the beneficial effects of xylitol may last for several years after exposure to this polyol, no one who has ever consumed xylitol would be eligible to take part in such a clinical trial!

\section{References}

Mäkinen KK: An end to crossover designs for studies on the effect of sug ar substitutes on caries? Caries Res 2009;43:331-333.

-Van Loveren C: Sugar alcohols: what is the evidence for caries-preventive and caries-therapeutic effects? Caries Res 2004;38:286-293.

\section{Dr. C. Dawes}

Department of Oral Biology, Faculty of Dentistry

University of Manitoba, 780 Bannatyne Avenue

Winnipeg, MB, R3E 0W2 (Canada)

Tel. +1 204789 3512, Fax +1 2047893943

E-Mail Colin_Dawes@umanitoba.ca
Caries Res 2010;44:170
DOI: $\underline{10.1159 / 000314070}$

\section{Reply to Professor Dawes}

\section{K.K. Mäkinen}

Institute of Dentistry, University of Turku, Turku, Finland

The objective of my 'Current Topic' piece was to question the automatic reliance on the so-called crossover study designs in clinical trials. The experience obtained from investigations with xylitol was used as an example. I still believe that 'blind' adherence to such a design may in some instances lead to erroneous conclusions, especially in cases where persisting long-term effects, such as those occasioned by antibiotics and fluorides, may prevail.

In line with the above objective of my writing, I found no reason to cite the review article of Dr. van Loveren; the objective was my concern regarding the crossover practice. Furthermore, excluding my own literature reviews, nearly 20 scientifically valid review articles on the dental effects of sugar alcohols have been published during the past 20 years. Several editorials and 'briefing papers' have also appeared. Most of them do not share the concept that all sugar alcohols exert exactly the same effects on dental caries. Instead, the possibility of more selective, specific polyol effects on oral biological processes has received support. Citing Dr. van Loveren's article would have obliged me to also list all of the other 20 reviews on this topic, which, as mentioned above, was not my aim. The issue of specific polyol effects on dental caries cannot be solved by writing reviews. As pointed out by Prof. Dawes, ' $\mathrm{t}$ ] he only truly relevant test is a proper clinical trial'. However, as we scientists so well know, no matter how solid the evidence for the success of a particular medical treatment, there will always be doubters. This is normal and human, and helps keep the scientific process vigorous and vigilant.

Kauko K. Mäkinen

Institute of Dentistry, University of Turku

Lemminkäisenkatu 2

FI-20520 Turku (Finland)

Tel. +358 2333 8227, Fax +358 23338201

E-Mail kauko.makinen@uusikaupunki.fi 